\title{
RESEARCH HIGHLIGHT Microbiota guides insulin trafficking in beta cells
}

\author{
Ozren Stojanović $\mathbb{D}^{1,2}$ and Mirko Trajkovski ${ }^{1,2,3}$ \\ Cell Research (2019) 29:603-604; https://doi.org/10.1038/s41422-019-0200-5
}

\begin{abstract}
Pancreatic beta cells produce insulin on intricate cellular assembly line, but the extent of the gut microbiota involvement was not clear. It emerges that fragments of bacterial cell wall released by lysozyme 1 in the intestinal lumen are necessary to keep the machinery running, acting on the cytosolic peptidoglycan receptor Nod1 in the beta cells to regulate the insulin trafficking and glucose tolerance.
\end{abstract}

Intestines are rich ecosystems where billions of bacteria from hundreds of species make their living by degrading food, mucus, and various metabolic byproducts of host and commensal microbes. Just as our external senses pick up clues from the environment and provoke physiological responses, the cues about bacterial environment inside intestines are sampled by many different "senses". Gut microbes communicate to organs and influence host physiology in a number of ways, ${ }^{1}$ however, little is known to which extent they can regulate the insulin trafficking. Bacterial metabolites, such us short-chain fatty acids, can enter circulation and regulate insulin secretion through an indirect signaling cascade that involves the vagus nerve. ${ }^{2}$ A recent study published in Cell Research by Zhang et al. ${ }^{3}$ reveals a cascade of interactions involving structural molecules of the gut microbiota, with the insulin-producing machinery of the beta cells, thus providing comprehensive direct link to the repertoire of microbiota-host communication.

Insulin is released from pancreatic beta cells in response to increased glucose levels after meal to promote nutrient uptake and energy storage. Islet beta cells produce insulin by stepwise processing of single polypeptide chain preproinsulin, which is translated and converted to proinsulin on the rough endoplasmatic reticulum adjacent to nucleus (Fig. 1). Still in the perinuclear region, proinsulin is sorted through membranes and vesicles of the Golgi network into secretory granules, also known as dense core vesicles (DCVs). DCVs then travel through the cytoplasm on microtubules, maturing along the way by vesicle fusion, cargo processing and conversion of proinsulin to insulin, until they reach vicinity of the plasma membrane. There, the secretory granules are ready to fuse with the membrane and release insulin in a dosedependent response to blood glucose.

Zhang et al. $^{3}$ discovered that in mice lacking intestinal microbiota, insulin DCVs remain co-localized with immature proinsulin DCVs around nucleus, in contrast to cytoplasmic distribution of mature DCVs seen in conventional mice. Insulin (but not proinsulin) content was reduced in islets of germ-free (GF) mice. Colonization of GF mice by gut flora restored normal vesicle distribution and insulin content. How do bacteria from gut guide insulin vesicles in beta cell cytoplasm? The authors suspected that peptides derived from bacteria might find their way to the beta cells where they would be recognized. Indeed, in beta cells lacking Nod1 (one of two known cytosolic receptors of peptidoglycan from bacterial cell wall), insulin and proinsulin vesicles were co-localized around the nucleus, much like in GF mice. Upon binding a peptidoglycan fragment, Nod1 recruits Rip2, its downstream adaptor. Rip2 knockout (KO) mice also lacked proper cytoplasmic diffusion of insulin vesicles, and so did $\mathrm{KO}$ mice for Rab1a, the next player in this triad. The authors demonstrate that Rab1a directs Nod1 and Rip2 to the insulin vesicles. Presence of all three components was necessary for proper cellular localization of the insulin vesicles, and for optimal insulin release upon glucose stimulation. The movement of the secretory granules along the microtubules may depend on kinesin-1, 4 a molecular motor protein previously reported to be linked with Rab1a. This may suggest that Nod1 ligands direct the cytosolic distribution of the DCVs by promoting their movement along the microtubules. Determining to which extend Nod1 ligands are involved in this, and in the remaining steps of the secretory granule exocytosis pends further investigation.

Mucus secreted by the gut shields the intestinal epithelial cells from contact with most microbes. Localized deeply in the intestinal crypts (from where the gut epithelium is constantly renewed), Paneth cells secrete antimicrobial peptides to the intestinal lumen that guard against bacterial intruders who happen to come too close. Among these peptides is the intestine-abundant lysozyme 1 (Lyz1), which hydrolyzes peptidoglycan in the bacterial cell wall, releasing fragments that can enter circulation. The study shows that conventional mice lacking Lyz1 have defective insulin trafficking and diminished insulin content and release, demonstrating that for efficient functioning, beta cells must be presented with structural components of commensal bacteria. Intriguingly, Nod1 in beta cells is sensitive to some peptidoglycan types-and therefore some types of bacteria-but not to the others. Inoculation of GF mice with Lactobacillis plantarum dispersed insulin vesicles, but with Lactococcus lactis (a different type of peptidoglycan) kept vesicles perinuclear. Though it seems that Nod1 system is not highly selective between particular species, but rather peptidoglycan types, it remains to be determined whether large-scale alterations in microbiome, associated with diverse physiological perturbations such us high-fat diet, could augment or reduce insulin production in this way. Supplementing bacteria-derived peptidoglycan to GF and Lyz1 KO mice restored efficient insulin dispersion, increased insulin content and potentiated glucose-stimulated insulin release in their beta cells; however, no such effects were observed in cultured beta cells or islets from WT mice. On the other hand, it was reported that high-fat diet increases circulating levels of Nod1 ligands that activate, through Nod1, proinflammatory macrophages in adipose tissue and induce insulin

\footnotetext{
${ }^{1}$ Department of Cell Physiology and Metabolism, Faculty of Medicine, Centre Médical Universitaire, University of Geneva, 1211 Geneva, Switzerland; ${ }^{2}$ Diabetes Centre, Faculty of Medicine, University of Geneva, 1211 Geneva, Switzerland and ${ }^{3}$ Institute of Genetics and Genomics in Geneva (IGE3), University of Geneva, 1211 Geneva, Switzerland Correspondence: Mirko Trajkovski (Mirko.Trajkovski@unige.ch)
}

Published online: 2 July 2019 


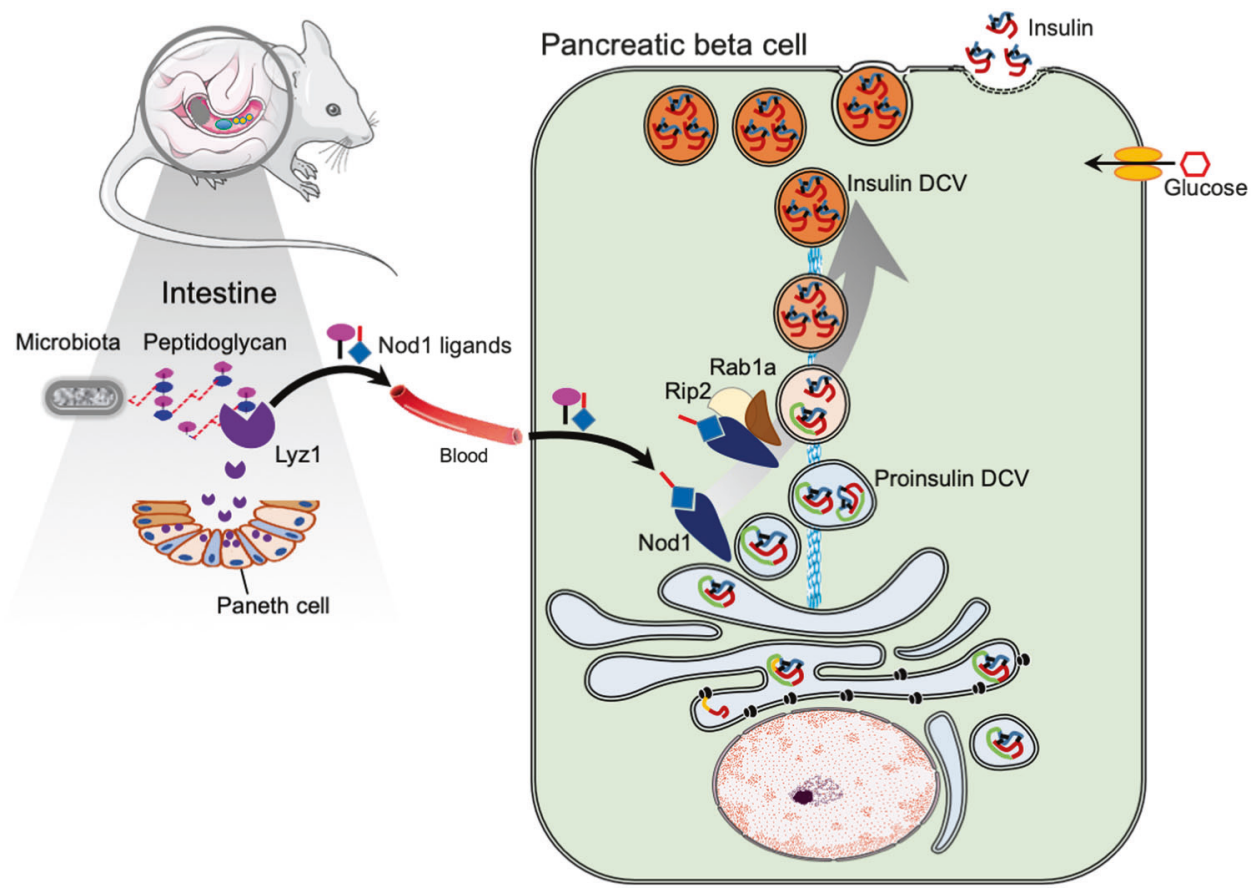

Fig. 1 Gut microbiota-derived signals to the beta cells are necessary for proper insulin trafficking. The intestinal lysozyme Lyz1 enables bacteria-derived peptidoglycans to reach the pancreatic beta cells through the circulation. In the pancreas, these ligands act on Nod1, enabling it to interact with its downstream adaptor Rip2 and with the small GTPase Rab1, necessary for maturation and cellular localization of the insulin vesicles, and for optimal insulin release upon glucose stimulation. Some of the items used to generate the figure were obtained from Servier Medical Art

resistance. ${ }^{5}$ If changing circulating levels of Nod1 ligands can alter Nod1 signaling in immune cells, it may be worth investigating whether similar mechanisms operate in beta cells during obesity.

What could be the physiological rationale for this microbiotainsulin interaction remains puzzling. It is known that absence or depletion of gut microbiota (and respectively Nod1 ligands) increases insulin sensitivity, especially in fat. ${ }^{6}$ Consistent with this, global double Nod1/Nod $2^{-1-}$ mice (Nod1 is ubiquitously expressed) have improved insulin sensitivity, while Nod1 ligands promote insulin resistance in peripheral tissues such as liver and fat. $^{7}$ Global Nod1 KO mice show normal glucose tolerance ${ }^{8}$ due to the lower glucose-stimulated insulin secretion from the beta cells, in agreement with the findings shown here using the beta cellspecific Nod1 KO mice. Therefore, it may be that Nod1 ligand- elicited signaling in the pancreatic beta cells is a mechanism to counterbalance the increased insulin demand associated with increased levels of microbial products upon colonization.

\section{REFERENCES}

1. Schroeder, B. O. \& Backhed, F. Nat. Med. 22, 1079-1089 (2016).

2. Perry, R. J. et al. Nature 534, 213-217 (2016).

3. Zhang, Q. et al. Cell Res. 29, 516-532 (2019).

4. Varadi, A., Tsuboi, T., Johnson-Cadwell, L. I., Allan, V. J. \& Rutter, G. A. Biochem. Biophys. Res. Commun. 311, 272-282 (2003).

5. Chan, K. L. et al. Cell Rep 18, 2415-2426 (2017).

6. Suarez-Zamorano, N. et al. Nat. Med. 21, 1497-1501 (2015).

7. Schertzer, J. D. et al. Diabetes 60, 2206-2215 (2011).

8. Amar, J. et al. EMBO Mol. Med. 3, 559-572 (2011). 